\title{
Interfacial progressive gravity waves in a two-layer shear flow
}

\author{
D. I. Pullin \\ Department of Mechanical Engineering, University of Queensland, St. Lucia, Queensland, 4067 Australia \\ R. H. J. Grimshaw \\ Department of Mathematics, University of Melbourne, Parkville, Victoria, 3052 Australia
}

(Received 15 November 1982; accepted 23 March 1983)

\begin{abstract}
Nonlinear interfacial gravity waves in a two-layer Boussinesq fluid are studied. In a previous paper, nonlinear waves on a vortex sheet separating two layers, each of constant density and velocity were considered. In the present paper the basic flow model consists of a constant vorticity upper layer bounded by a rigid surface and an irrotational lower layer of infinite depth with continuity of the unperturbed velocity at the density interface. Numerical solutions obtained from an exact formulation in terms of a complex-valued integral equation for the shape and local vortex-sheet strength of the wave profile are compared with results from a second-order Stokes expansion. It is found that the wave of maximum amplitude displays different geometrical features depending on the unperturbed flow parameters. These include waves containing an Sshaped section, waves with cusped crests and surface-constrained waves with long flat crests. Wave integral properties calculated, including the flux of momentum and energy in the wave propagation direction, showed monotonic variation with increasing wave amplitude.
\end{abstract}

\section{INTRODUCTION}

It is well known that density stratified shear flows can support wave propagation. For instance, large-amplitude waves are commonly observed on the oceanic thermocline, or on atmospheric inversion layers. These waves have their largest amplitudes in regions where the basic density and shear flow profiles change rapidly. Hence in a previous pa$\operatorname{per}^{1}$ (henceforth denoted by I) we studied nonlinear interfacial progressive waves in a two-layer model in which both the density and unperturbed shear flow velocity were constant within each layer but were discontinuous at the interface separating the layers. The main simplifying feature of this model is that the perturbed flow in each layer is irrotational, and consequently the equations describing the nonlinear progressive waves could be reduced to a complex-valued nonlinear integral equation for the wave shape at the interface. This equation was then solved numerically and the results compared to those obtained by an analytical thirdorder Stokes expansion of an equivalent formulation in terms of the stream function.

The principal purpose of the present paper is to replace the discontinuous shear flow in I with a model in which the unperturbed shear flow has constant velocity in the lower layer, is continuous at the interface and has constant vorticity in the upper layer. This model (as will be shown) retains the feature that the perturbed flow in each layer is irrotational, and again allows the problem to be reduced to a complex-valued nonlinear integral equation for the wave shape of the interface. In the absence of buoyancy effects it has been used previously in the study of both unsteady ${ }^{2}$ and quasisteady periodic ${ }^{3}$ shear flows. For the present application, as well as being a more realistic representation of observed gravity/shear flow waves, the model has the advantage that the unperturbed flow is stable to infinitesimal disturbances for all wavenumbers, unlike that used in I, which because of the interfacial mean velocity discontinuity, exhibited high wavenumber Kelvin-Helmholtz instability. As in I, the density profile is that for a two-layer fluid (i.e., the density is constant in each layer), the Boussinesq approximation is used and consistently with this the upper boundary for the upper layer is taken to be rigid; the lower layer is assumed to be infinitely deep.

Several studies of nonlinear progressive waves have appeared in the literature, and we refer to I for a relevant discussion. Most of these, however, do not consider the effect of a shear flow; and hence, as in I, we focus our attention on this aspect of our results. By a combination of numerical solutions and small-amplitude Stokes expansions, we derive the wave shape at the interface; the wave phase speed and other wave properties such as the wave momentum, kinetic and potential energies, radiation stress and energy flux are also derived. Since completion of our work in I, the related work of Saffman and Yuen ${ }^{4}$ has been published. They consider nonlinear interfacial waves on a vortex sheet separating two semi-infinite layers, each of constant density and with constant basic velocity. In contrast to I, they do not make the Boussinesq approximation and emphasize the parametric dependence of the waves on the basic shear; whereas in I, we were more concerned with establishing wave properties as a function of wave amplitude. Also in I, we explored the effect of a finite depth to the upper layer. Where there is an overlap between Saffman and Yuen and I (for instance, in the weakly nonlinear analytical theory) we are in agreement.

In Sec. II the problem is defined and the Stokes expansion is obtained to second order. Then in Sec. III the nonlinear complex-valued integral equation formulation is developed, and a modified version of the Fourier-expansion solution procedure described in I is used to allow an improved calculation of large-amplitude waves. The numerical and analytical results for the wave profiles and speed are 
discussed in Sec. IV, while other wave integral properties are defined and evaluated in Sec. V.

\section{THE CONSTANT SHEAR MODEL}

\section{A. Formulation}

We shall study two-dimensional progressive waves at the interface between two incompressible and inviscid fluids. In coordinates $(x, y)$, the periodic wave of wavelength $\lambda$ propagates in the $x$ direction and gravity $g$ acts in the negative $y$ direction. The fluid densities are $\rho_{1}$ and $\rho_{2}$ with $\rho_{2}>\rho_{1}$, where subscripts 1 and 2 refer to fluid properties above and below the interface, respectively. The $x$ axis is the mean level of the wave, and the flow is bounded by horizontal planes at $y=d_{2}$ and $y=d_{1}$. The basic unperturbed flow in the twolayer model is described by the velocity field

$$
\begin{aligned}
& u_{1}=\bar{u}_{1}-\omega_{1} y, \quad v=0 ; \quad d_{1} \geqslant y \geqslant 0, \\
& u_{2}=\bar{u}_{2}, \quad v=0 ; \quad 0 \geqslant y \geqslant-d_{2},
\end{aligned}
$$

where the $\bar{u}_{j}, j=1,2$ are constant velocities and $\omega_{1}$ is the constant vorticity in the upper layer. Note that in (1a) and (1b) we have included a finite mean velocity jump across the interface for greater generality, and also to provide a comparison with the formulation of I. However in Sec. III and subsequently, we shall put $\bar{u}_{1}=\bar{u}_{2}=0$, and also let $d_{2} \rightarrow \infty$. In the presence of a finite-amplitude wave, we assume that the vorticity in the upper layer remains uniform and equal to $\omega_{1}$ and that the lower layer remains irrotational. Thus the perturbed flow satisfies the Helmholtz theorem for two-dimensional inviscid flow. We do not discuss the detailed wave formation process, but note that the constant $\omega_{1}$ model is consistent with wave generation by the action of conservative forces on the basic flow at infinity.

Using $\omega_{2}=0$ to denote the vorticity in the lower fluid, the flow in the presence of the wave may be described by

$$
\begin{aligned}
u_{j} & =\bar{u}_{j}-\omega_{j} y+\hat{u}_{j}, \quad v_{j}=\hat{v}_{j}, \quad j=1,2, \\
\psi_{j} & =\bar{u}_{j} y-\frac{1}{2} \omega_{j} y^{2}+\hat{\psi}_{j}, \quad j=1,2, \\
\phi_{j} & =\hat{\phi}_{j}, \quad j=1,2,
\end{aligned}
$$

where the careted quantities are those associated with perturbation of the basic flow. Since $\nabla^{2} \psi_{j}=-\omega_{j}$ in each region where $\psi_{j}$ is the stream function then $\hat{\psi}_{j}$ and the perturbation velocity potential $\hat{\phi}_{j}$ are those for an irrotational field with $\hat{u}_{j}=\partial \hat{\phi}_{j} / \partial x=\partial \hat{\psi}_{j} / \partial y$ and $\hat{v}_{j}=\partial \hat{\phi}_{j} / \partial y=-\partial \hat{\psi}_{j} / \partial x$. In the reference frame of the basic flow (1), we denote the perturbed interface shape by $y=\eta(x, t)$ with zero mean and let the phase speed be $c$.

By choosing $\left.\hat{\psi}_{1}\right|_{y=d_{1}}=0$ and utilizing a transformation of the form $x^{\prime}=x-c t$ to a reference frame moving with the wave, it may easily be shown that the boundary conditions to be satisfied on the interface $y=\eta$ for the existence of nonlinear progressive waves are

$v_{1}=v_{2}=0 \quad$ on $y=d_{1},-d_{2}$,

$-\left(c-\bar{u}_{j}\right) \eta-\frac{1}{2} \omega_{j} \eta^{2}+\left.\hat{\psi}_{j}\right|_{\eta}=Q_{j}=\mathrm{const}, \quad j=1,2$,

$p_{1}=p_{2}$

In the kinematic condition $(3 \mathrm{~b}), Q_{j}$ is the excess $x$-wise vol- ume flux in layer $j$ given by

$$
\begin{aligned}
-Q_{1} & =\left\langle\int_{\eta}^{d_{1}} u_{1} d y\right\rangle-\left(\bar{u}_{1} d_{1}-\frac{1}{2} \omega_{1} d_{1}^{2}\right) \\
& =\left\langle\int_{\eta}^{d_{1}} \hat{u}_{1} d y\right\rangle+\frac{1}{2} \omega_{1}\left\langle\eta^{2}\right\rangle, \\
Q_{2} & =\left\langle\int_{-d_{2}}^{\eta} y_{2} d y\right\rangle-\left(\bar{u}_{2} d_{2}+\frac{1}{2} \omega_{2} d_{2}^{2}\right) \\
& =\left\langle\int_{-d_{2}}^{\eta} \hat{u}_{2} d y\right\rangle-\frac{1}{2} \omega_{2}\left\langle\eta^{2}\right\rangle,
\end{aligned}
$$

where the average over a wavelength is given by

$$
\langle\rangle=\frac{1}{\lambda} \int_{0}^{\lambda}(1) d x \text {. }
$$

In the dynamic condition $(3 \mathrm{c})$ the pressures $p_{j}$ at a general point in layer $j$ are given by the modified Bernoulli equation for constant vorticity rotational progressive-wave-like flow

$p_{j} / \rho_{j}+\frac{1}{2} \hat{u}_{j}^{2}+\frac{1}{2} \hat{v}_{j}^{2}-\left(c-\bar{u}_{j}\right) \hat{u}_{j}+g y-\omega_{j}\left(y \hat{u}_{j}-\hat{\psi}_{j}\right)=L_{j}$,

where the $L_{j}, j=1,2$ are (different) Bernoulli constants within each layer. Note that in establishing (6) we have utilized $\partial / \partial t=-c \partial / \partial x$ to eliminate the $\partial \hat{\phi}_{j} / \partial t$ term in the time-dependent Bernoulli equation.

\section{B. Solution by the Stokes expansion}

The analytical solution through the Stokes expansion in the wave amplitude proceeds as in I, except that here we must include the extra terms due to the presence of rotational flow in the upper layer. Thus we expand $\eta$ and $\hat{\psi}_{j}$ as

$$
\begin{aligned}
& \eta=\sum_{-\infty}^{\infty} \eta_{n} e^{i n k(x-c t)} \\
& \hat{\psi}_{1}=\sum_{-\infty}^{\infty} a_{n} \frac{\sinh \left[n k\left(d_{1}-y\right)\right]}{\sinh \left(n k d_{1}\right)} e^{i n k(x-c t)} \\
& \hat{\psi}_{2}=\sum_{-\infty}^{\infty} b_{n} \frac{\sinh \left[n k\left(d_{2}+y\right)\right]}{\sinh \left(n k d_{2}\right)} e^{i n k(x-c t)}
\end{aligned}
$$

where $k=2 \pi / \lambda, \eta_{-n}=\eta_{n}^{*}, a_{-n}=a_{n}^{*}$ and $b_{-n}=b_{n}^{*}$, where ${ }^{*}$ denotes the complex conjugate. If the wave amplitude $\Delta$ is given by

$$
2 \Delta=\eta_{\max }-\eta_{\min },
$$

then substituting (7) into (3) and using (6) we find to the first order in $\Delta$

$$
\begin{aligned}
& \eta_{1}=\Delta / 2, \\
& a_{1}=\left(c-\bar{u}_{1}\right) \eta_{1}, \quad b_{1}=\left(c-\bar{u}_{2}\right) \eta_{1} .
\end{aligned}
$$

The first order dispersion relationship is for $\omega_{2}=0$ is

$g\left(\rho_{2}-\rho_{1}\right)-\rho_{2}\left(c-\bar{u}_{2}\right)^{2} k S_{2}$

$$
-\rho_{1}\left(c-\bar{u}_{1}\right)^{2} k S_{1}-\rho_{1} \omega_{1}\left(c-\bar{u}_{1}\right)=0,
$$

where $S_{j}=\operatorname{coth}\left(k d_{j}\right)$. For our case of principal interest $\bar{u}_{1}=\bar{u}_{2}=0$ so that the zeroth-order phase speed is given by

$c_{0}=\frac{-\rho_{1} \omega_{1} \pm\left[\rho_{1}^{2} \omega_{1}^{2}+4 g k\left(\rho_{2}-\rho_{1}\right)\left(\rho_{1} S_{1}+\rho_{2} S_{2}\right)\right]^{1 / 2}}{2 k\left(\rho_{1} S_{1}+\rho_{2} S_{2}\right)}$

For given $\omega_{1}$, there are always two solutions; an upper branch in which the wave moves in the same direction as the 
undisturbed fluid in the upper layer and a lower branch in which the wave motion is opposed to the upper layer motion. Thus, unlike the case treated in $I\left(\omega_{1}=0, \bar{u}_{1} \neq 0\right)$, the basic flow (1) is first order stable to small disturbances for all finite $k$ so that possible instabilities must be asociated with nonlinear finite amplitude effects.

The Stokes expansion may be readily taken to higher order. Thus to second order we find that

$$
\begin{aligned}
\eta_{2}= & k F \eta_{1}^{2}, \\
G F= & \frac{1}{2}\left(\rho_{2}\left(c-\bar{u}_{2}\right)^{2}\left(3 S_{2}^{2}-1\right)-\rho_{1}\left(c-\bar{u}_{1}\right)^{2}\left(3 S_{1}^{2}-1\right)\right. \\
& \left.\quad-\left(\omega_{1} / k\right)\left[\left(c-\bar{u}_{1}\right)\left(3 S_{1}+1 / S_{1}\right)+\omega_{1} / k\right]\right\} \\
G= & \frac{\rho_{2}\left(c-\bar{u}_{2}\right)^{2}}{S_{2}}+\frac{\rho_{1}\left(c-\bar{u}_{1}\right)^{2}}{S_{1}} \\
b_{2}= & \left(c-\bar{u}_{2}\right)\left(\eta_{2}-k \eta_{1}^{2} S_{2}\right), \\
a_{2}= & \left(c-\bar{u}_{1}\right)\left(\eta_{2}-k \eta_{1}^{2} S_{1}\right)+\frac{1}{2} \omega_{1} \eta_{1}^{2} .
\end{aligned}
$$

The second-order dispersion relationship is

$$
\begin{aligned}
g\left(\rho_{2}-\right. & \left.\rho_{1}\right)-\rho_{2}\left(c-\bar{u}_{2}\right) k S_{2}-\rho_{1}\left(c-\bar{u}_{1}\right)^{2} k S_{1}-\rho_{1} \omega_{1}\left(c-\bar{u}_{1}\right) \\
= & k\left|\eta_{1} k\right|^{2}\left\{-2 F^{2} G+2 \rho_{2}\left(c-\bar{u}_{2}\right)^{2} S_{2}\left(S_{2}^{2}-2\right)\right. \\
& +2 \rho_{1}\left(c-\bar{u}_{1}\right)^{2} S_{1}\left(S_{1}^{2}-2\right) \\
& +\left(\omega_{1} \rho_{1} / k\right)\left[2\left(c-\bar{u}_{1}\right)\left(S_{1}^{2}-1\right)\right. \\
& \left.\left.+\left(\omega_{1} / 2 k\right)\left(S_{1}+1 / S_{1}\right)\right]\right\} .
\end{aligned}
$$

\section{INTEGRAL EQUATION FORMULATION}

In order to reduce the number of independent parameters we now consider only the case of major physical interest $\bar{u}_{1}=\bar{u}_{2}=0$ in the deep water limit $d_{2} \rightarrow+\infty$. Using $u^{\prime}=u-c, v^{\prime}=v$ to denote velocities in the reference frame at rest with respect to the wave (6) may be written on $y=\eta$ as

$$
\begin{aligned}
& p_{1} / \rho_{1}+\frac{1}{2} u_{1}^{\prime 2}+\frac{1}{2} v_{1}^{\prime 2}+g \eta=L_{1}-\omega_{1} Q_{1}+\frac{1}{2} c^{2}, \\
& p_{2} / \rho_{2}+\frac{1}{2} u_{2}^{\prime 2}+\frac{1}{2} v_{2}^{\prime 2}+g \eta=\frac{1}{2} c^{2} .
\end{aligned}
$$

In obtaining (14) we have taken $L_{2} \rightarrow 0$ for $d_{2} \rightarrow-\infty$ (see Sec. V) and have used (3) to eliminate $\left.\widehat{\psi}_{j}\right|_{\eta}$. Since Eqs. (14) are of the same form as for an irrotational two-layer model the formulation of the boundary conditions in terms of a complex velocity field used in I may be modified for use in the present model. Briefly, we introduce quantities $U, V, C, X$, and $Y$ as dimensionless forms of $u^{\prime}, v^{\prime}, c, x$, and $y$, respectively, based on the length scale $\lambda / \pi$ and time scale $(\lambda / \pi \alpha g)^{1 / 2}$ where $\alpha=\left(\rho_{2}-\rho_{1}\right) /\left(\rho_{2}+\rho_{1}\right)$ is the Boussinesq parameter. Describing here the interface shape by the complex function $z(a)=X_{c}(a)+i Y_{c}(a)$, where $a$ is an interfacial parameter and introducing the Boussinesq limit $\alpha \rightarrow 0$, the boundary conditions on the interface may be written as in I,

$$
\gamma\left(\frac{\partial z^{*}}{\partial a}\right)^{-1}(U-i V)_{p}+2 Y_{c}+\Lambda_{1}-\Omega \hat{Q}_{1}=0 .
$$

In (15) $\gamma=\partial \Gamma / \partial a, \Gamma$ is the dimensionless cumulative circulation along the interface (i.e., the integral of the tangential velocity difference), $\Lambda_{1}, \Omega$, and $\hat{Q}_{1}$ are dimensionless forms of $L_{1}, \omega_{1}$, and $Q_{1}$, respectively, and $(U-i V)_{p}$ refers to the mean of the generally unequal fluid velocities on either side of the interface.

\section{A. The complex velocity field}

The complex velocity field at a general point $Z=X+i Y$ in either fluid may be expressed in terms of an appropriately chosen distribution of singularities along a single interface wavelength $\pi \geqslant a \geqslant 0$. Thus we write

$$
\begin{aligned}
U-i V= & \frac{1}{2 \pi i} \int_{0}^{\pi}\left\{\cot \left(Z-z^{\prime}\right)\left[\gamma^{\prime}-\Omega Y_{c}^{\prime}\left(\frac{\partial z^{\prime}}{\partial a^{\prime}}\right)\right]\right. \\
& \left.-\cot \left(Z-z^{*}-2 i D\right)\left[\gamma^{\prime}-\Omega Y_{c}^{\prime}\left(\frac{\partial z^{* \prime}}{\partial a^{\prime}}\right)\right]\right\} d a^{\prime} \\
& -A \Omega Y,
\end{aligned}
$$

where $A=1$, for $Z$ in the upper fluid layer and $A=0$ for $Z$ in the lower fluid, and where dashed quantities are all functions of $a^{\prime}$. The integral term in (16) is an analytic function of $Z$ and thus represents an irrotational velocity field while the second term represents the rotational field in the upper layer. The second term in the first square bracket is a distribution of source and vortex singularities of strength chosen so that the resulting velocity discontinuity at the interface just cancels that due to the rotational field. Hence the overall velocity jump is purely tangential and due to the distribution of vortex singularities of strength $\gamma$ given by the first term in the integral. The second term in curly brackets is a mirror image of the singularity distribution in the upper rigid surface $Y=D=d_{1} \pi / \lambda$ introduced so as to satisfy (1a). By expanding the integral in powers of $e^{-|Y|}$ for $Z$ far removed from the interface it may be readily shown that the basic flow (1) is approached asymptotically as $Y \rightarrow \pm \infty$ provided that

$$
\int_{0}^{\pi} \gamma\left(a^{\prime}\right) d a^{\prime}=0
$$

and also that the mean interface level is zero.

Evaluating (16) at a point on the interface (in which case the first term is a Cauchy Principal Value integral) and substituting into (15) leads to an integral equation for $Y_{c}(a)$ and $\gamma(a)$. The closure conditions determining $C$ and the single parameter $A_{1}-\Omega \widehat{Q}_{1}$ are the zero mean interface level and wave amplitude restraints

$$
\int_{0}^{\pi} X_{c} \frac{\partial X_{c}}{\partial a} d a=0, \quad Y_{c_{\max }}-Y_{c_{\min }}=2 \delta
$$

where $\delta=\Delta \pi / \lambda$.

\section{B. Numerical solution}

A numerical solution of the integral equation was obtained by expanding $z(a)$ and $\gamma(a)$ Fourier series, viz.,

$$
\begin{aligned}
& z(a)=a+\sum_{n=0}^{N-1}\left[A_{n} \sin (2 n a)+i B_{n} \cos (2 n a)\right], \\
& \gamma(a)=\sum_{n=1}^{N} E_{n} \cos (2 n a) .
\end{aligned}
$$

Implicit in (19) is the assumption that the wave shape is symmetrical about either the crest $(a=0)$ or trough $(a=\pi / 2)$. While a class of nonsymmetrical nonlinear waves may be possible we restrict attention to the symmetrical case for simplicity. If the $A_{n}$ are specified, substituting (19) into (18) and (15) and (16) evaluated at the $N+1$ points $a_{n}=n \pi /$ $(2 N), n=0 \cdots N$ (the numerical treatment of the integrals is as in I) leads to a closed system of $2 N+2$ nonlinear equations 
for the $2 N+2$ quantities $B_{n}, n=0 \cdots N-1, E_{n}, n=1 \cdots N$, $\Lambda_{1}-\Omega \widehat{Q}_{1}$ and $C$. Apart from slow convergence of (19) for steep waves however, this scheme suffers from the further disadvantage that it cannot deal with possible S-shaped waves in which $Y$ is a multiple valued function of $X$ along some portion of the interface. Thus we adopt an alternative scheme in which we introduce a set of chord length equations

$$
\begin{aligned}
\mid z\left(a_{n}\right) & -z\left(a_{n-1}\right) \mid \\
& -\epsilon \Delta a\left\{1+\frac{1}{2} \beta\left[\cos \left(2 a_{n}\right)+\cos \left(2 a_{n-1}\right)\right]\right\}=0,
\end{aligned}
$$$$
n=1 \cdots N
$$

where $\Delta a=\pi /(2 N), 1>\beta \geqslant 0$, is a specified parameter and $\epsilon$ is unknown. Equation (20) places restrictions on the separation of adjacent interface points corresponding to $a_{n-1}, a_{n}$ (e.g., for $\beta=0$ all chord lengths are equal), and $\beta$ is introduced to allow smaller point separation in regions of high interface curvature.

Satisfying (20) in addition to the other conditions increases the number of equations to $3 N+2$ with extra unknowns $A_{n}, n=1 \cdots N-1$ and $\epsilon$. The increased computational effort required to solve the larger system however is more than compensated for by both accelerated convergence of (19) for very steep waves and by the relaxation of the previous restriction to waves with single valued shape functions $Y_{c}(X)$.

The Newton-Raphson iterative method was used to solve the $3 N+2$ nonlinear equations. Families of solutions were obtained by fixing $\Omega$ and $D$ and by increasing $\delta$ in small increments until a value $\delta_{\max }$ was reached for which the iteration scheme diverged. This then defined the highest wave that could be obtained by the solution method. Calcu-

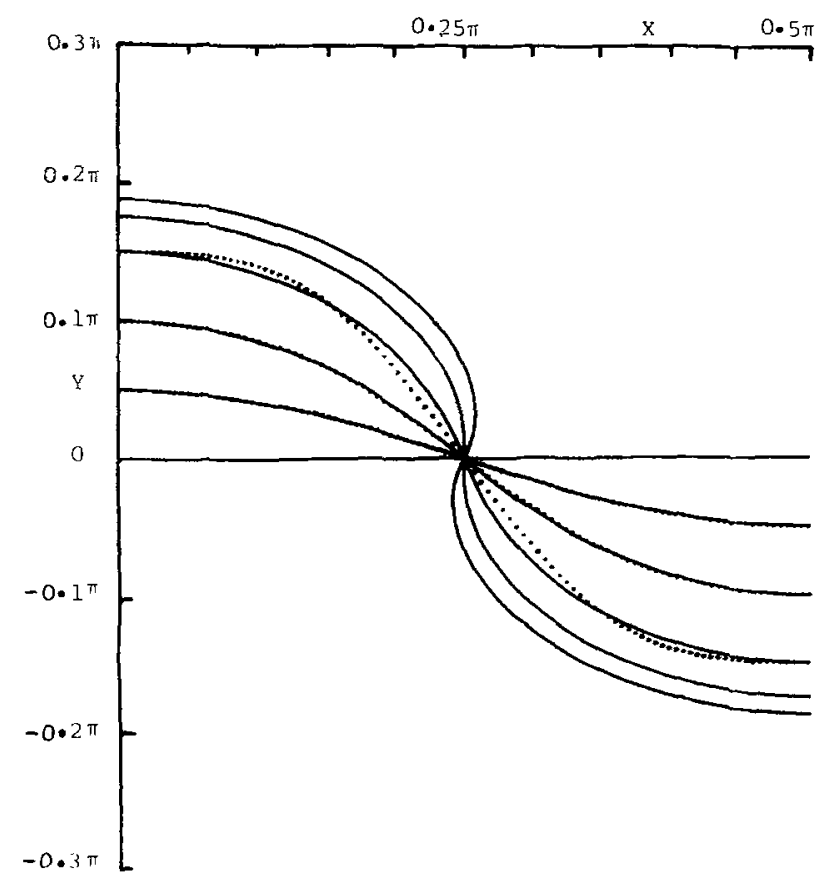

FIG. 1. Wave profiles, $D \rightarrow \infty, \Omega=0$. numerical, $\delta / \pi=0.05,0.10,0.15$, $0.1751,0.1875 ; \ldots$ Stokes expansion, $\delta / \pi=0.05,0.10,0.15$. lations were performed in single precision ( 32 bit) mode on a VAX $11 / 780$ machine with values of $N$ increasing from 30 to a maximum of 120 with increasing $\delta$. For each $D, \Omega$ set, some solutions including those near $\delta_{\max }$ were calculated to double precision ( 64 bit) accuracy, but it was found that this refinement lead to negligible changes (of order $10^{-6}$ or smaller) in significant quantities such as the wave profile shape or the integral properties, including $C$. Solutions were sought in which the ratio, say $R_{A}, R_{B}$, or $R_{E}$ of the highestorder coefficient in (19) to the largest coefficient was of order $10^{-7}$ and $10^{-14}$ in single and double precision modes respectively. This accuracy was not always achieved, however, due to slow convergence of $(19 \mathrm{~b})$ and in some cases $R_{A} \sim R_{B}$ $\sim O\left(10^{-7}\right), R_{F} \sim O\left(10^{-6}\right)$ were accepted as solutions in double precision mode.
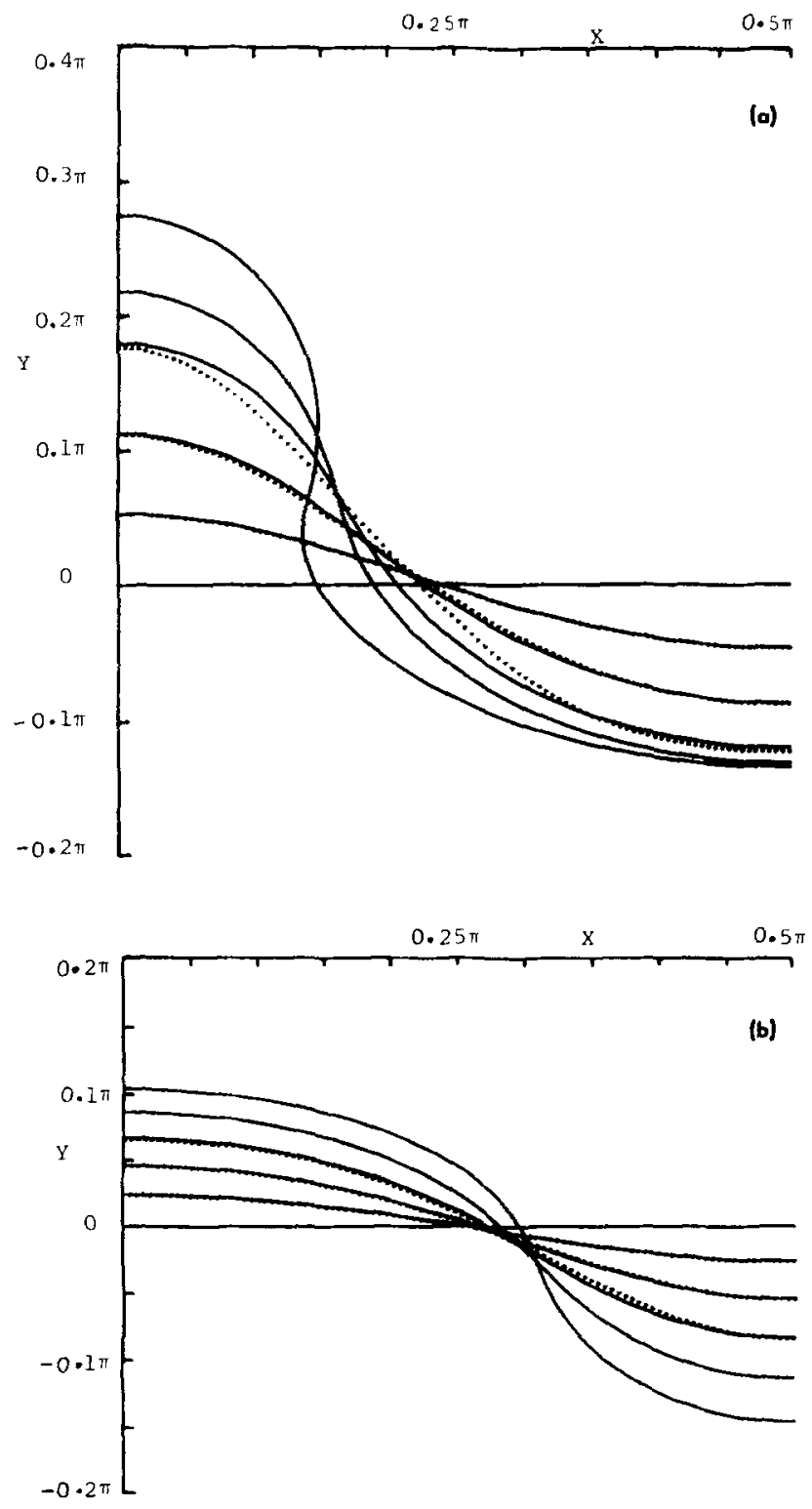

FIG. 2. (a) Wave profiles $D \rightarrow \infty, \Omega=-2 / \pi$, upper branch; - numerical, $\delta / \pi=0.05,0.10,0.15,0.175,0.205 ; \quad \cdots \quad$ Stokes expansion. $\delta /$ $\pi=0.05,0.10,0.15$. (b) Wave profiles, $D \rightarrow \infty, \Omega=-2 / \pi$, lower branch: -numerical, $\delta / \pi=0.025,0.05,0.075,0.10,0.125 ; \cdots$ Stokes expansion, $\delta /$ $\pi=0.025,0.05,0.075$. 


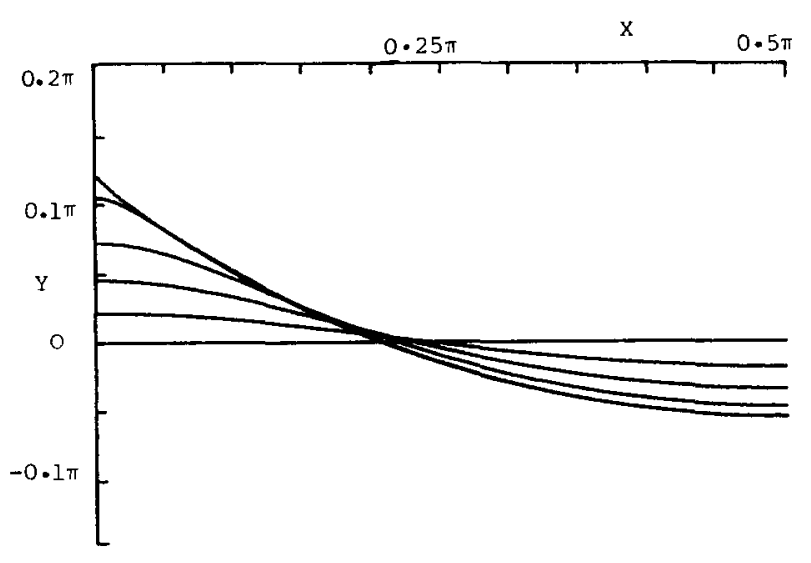

FIG. 3. Wave profiles, $D \rightarrow \infty, \Omega=-100 / \pi$, upper branch; - numerical, $\delta / \pi=0.02,0.04,0.05,0.08,0.0875$.

\section{RESULTS}

Values of $D=0.01 \pi$ with $\Omega=-20 / \pi,-100 / \pi$ and $D=0.1 \pi$ with $\Omega=-2 / \pi,-10 / \pi$, and $-20 / \pi$ were chosen as reasonably typical of the thermocline application, and also give similar values for the unperturbed velocity at the rigid surface $(-\Omega D)$ as were used in I for the irrotational model. In addition, cases for $D=\infty$ with $\Omega=0,-2 / \pi$ and $-100 / \Omega$ were studied, the first to test the modified Fourier series method against earlier results and the others to examine the effects of pure shear. Figures 1-6 show selected sets of half-wavelength profiles each with $\delta_{\max } \geqslant \delta>0$. The
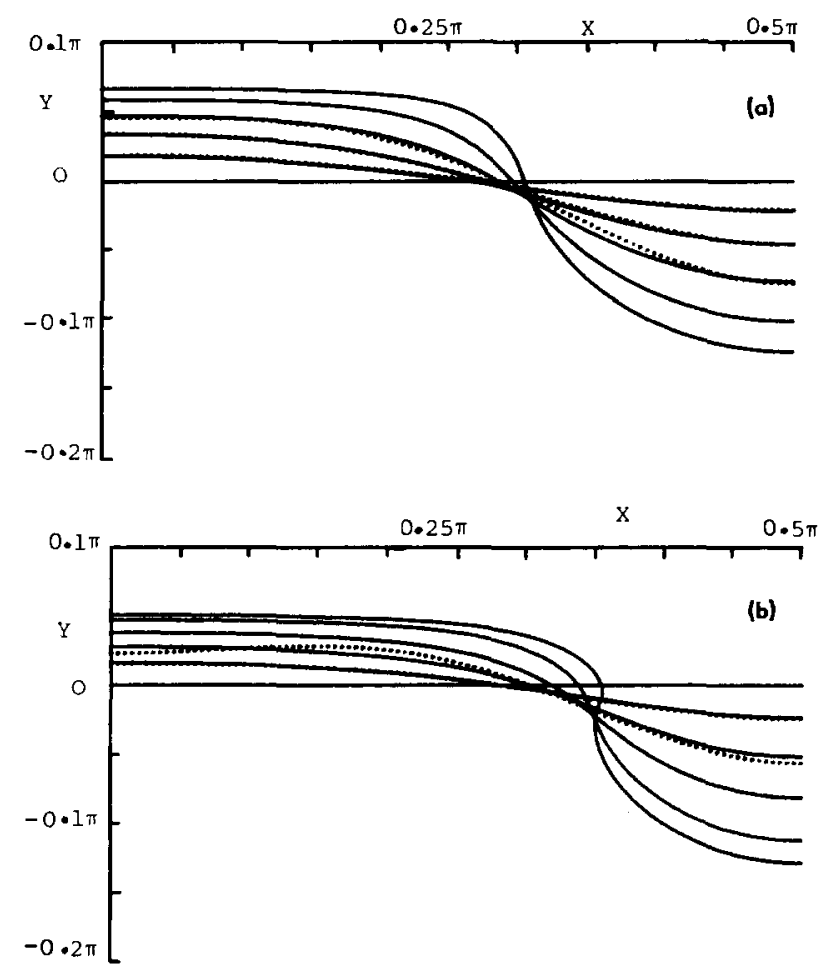

FIG. 4. (a) Wave profiles, $D / \pi=0.1, \Omega=-2 / \pi$, upper branch, - numerical, $\delta / \pi=0.2,0.4,0.6,0.8,0.95 \times 10^{-1} ;$...Stokes expansion, $\delta /$ $\pi=0.2,0.4,0.6 \times 10^{-1}$. (b) Wave profiles, $D / \pi=0.1, \Omega=-2 / \pi$, lower branch; - numerical, $\delta / \pi=0.2,0.4,0.6,0.8,0.9 \times 10^{-1} ; \cdots$ Stokes expansion, $\delta / \pi=0.2,0.4 \times 10^{-1}$.

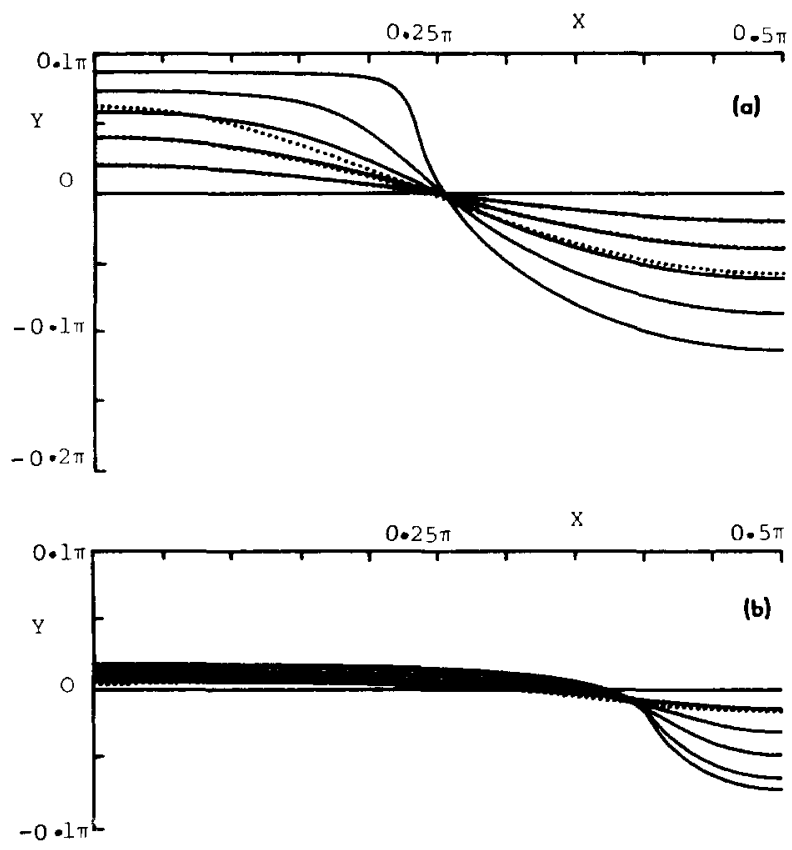

FIG. 5. (a) Wave profiles, $D / \pi=0.1, \Omega=-10 / \pi$, upper branch; - numerical, $\delta / \pi=0.2,0.4,0.6,0.8,1.0 \times 10^{-1}$. $\cdots$ Stokes expansion, $\delta /$ $\pi=0.2,0.4,0.6 \times 10^{-1}$. (b) Wave profiles, $D / \pi=0.1, \Omega=-10 / \pi$, lower branch; - numerical, $\delta / \pi=0.1,0.2,0.3,0.4,0.45 \times 10^{-1} ; \cdots$ Stokes expansion, $\delta / \pi=0.1 \times 10^{-1}$.

Stokes expansion profiles shown for comparison are those to second order from Sec. IIB except in Fig. 1 where thirdorder results from I are shown; Eqs. (12) shows that the order $\delta^{2}$ correction vanishes for this case. The wave profiles and other results for $D=\infty, \Omega=0$ agreed within $4-5$ digits with the calculations of I up to $\delta=0.1751 \pi$, where wave shape first develops a vertical section. The highest wave calculated with the present method for $\delta_{\max }=0.1875 \pi$ may be seen to develop a distinct $S$-section about the point of inflection.

In Figs. 2-3 the wave profiles base their antisymmetry about the $x$ axis because of shear in the upper half plane. The highest wave for $D \rightarrow \infty, \Omega=-2 / \pi$ (upper branch) in Fig.

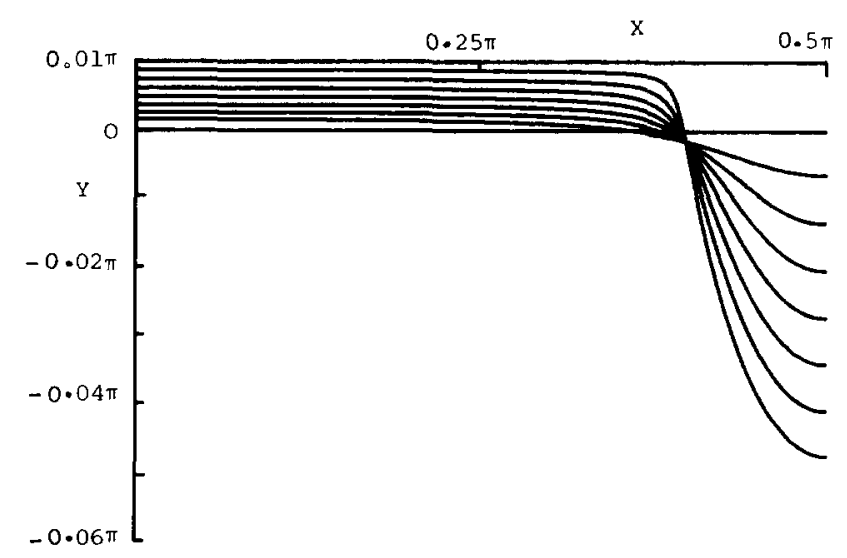

FIG. 6. Wave profiles, $D / \pi=0.01, \Omega=-100 / \pi$, upper branch; - numerical, $\delta / \pi=0.4,0.8,0.12,0.16,0.20,0.24,0.28 \times 10^{-2}$. 
2(a) exhibits an S-shaped section, but the corresponding lower branch solutions [Fig, 2(b)] shows a highest wave $\left(\delta_{\max }=0.125\right)$ with finite slope everywhere. Of other profile sets shown, only that of Fig. 4(b) shows an S-shaped highest wave, while those of Figs. 5(a) and 6 appear to be ceiling constrained. A quite different type of limiting solution is exhibited for the high shear case of Fig. 3 where the crest for the highest wave approaches a cusp similar to the Stokes limiting water wave solution. Generally however the wave shapes for finite $D$ are similar to corresponding cases with $\Omega=0$ (see I) indicating that moderate shear does not strongly affect the profile.

In claiming the S-shaped waves as solutions of Eqs. (15) and (16), we should comment that machine accuracy was not obtained for these cases. For instance, the $\delta_{\max }$ solution of Fig. 2(a) was obtained with $N=120$, and $R_{B} \sim O\left(10^{-7}\right)$, $R_{E} \sim O\left(10^{-6}\right)$ in double precision model indicating (at best) very slow convergence of (19). However, since S-shaped waves are almost certainly gravitationally unstable, the question of their existence is mainly academic. We also note that the present numerical method is not capable of computing a solution with a cusped crest since the wave slope at the crest is required to be zero. Thus the maximum amplitude solution of Fig. 3 was calculated with $N=50$ and $\beta=-0.99$ in (20) which strongly clusters points in the crest region.

Figures 7-12 show vector velocity field plots in the frame of reference at rest with respect to the wave. The arrows are of a length proportional to the velocity at the local downstream arrow end. Examination of the velocity field for the $\delta_{\max }$ wave in each case suggests the common appearance of one or more stagnation points along the interface profile so that the local fluid velocity equals the wave velocity in the

(a)

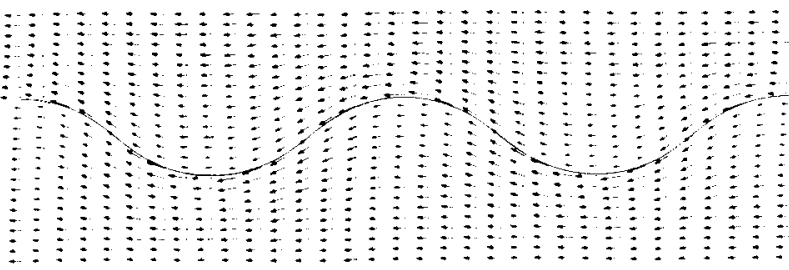

(b)

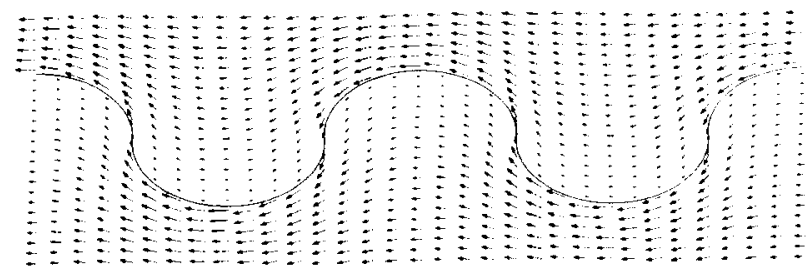

(c)

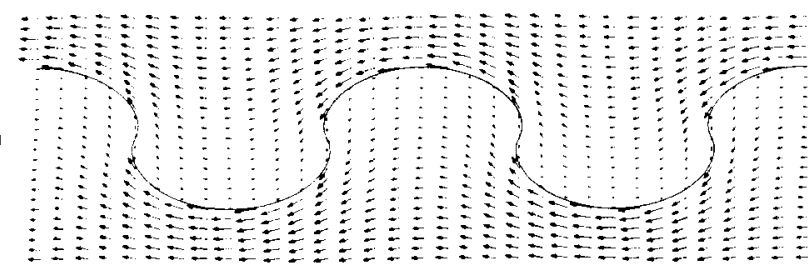

FIG. 7. Near interface velocity field in reference frame at rest with respect to the wave, $D \rightarrow \infty, \Omega=0$, (a) $\delta / \pi=0.10$, (b) $\delta / \pi=0.175$, (c) $\delta$ / $\pi=0.1875$.

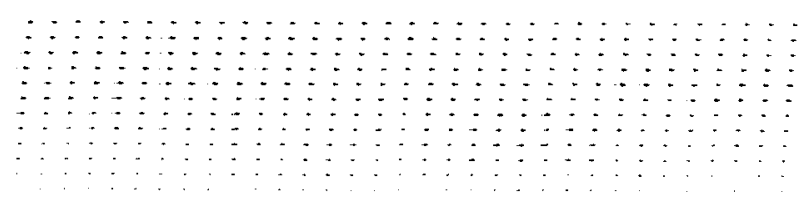

(a)

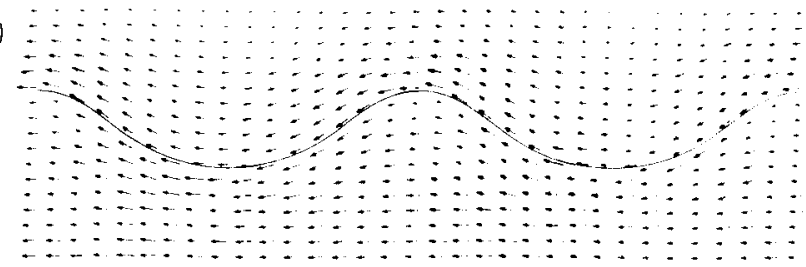

(b)
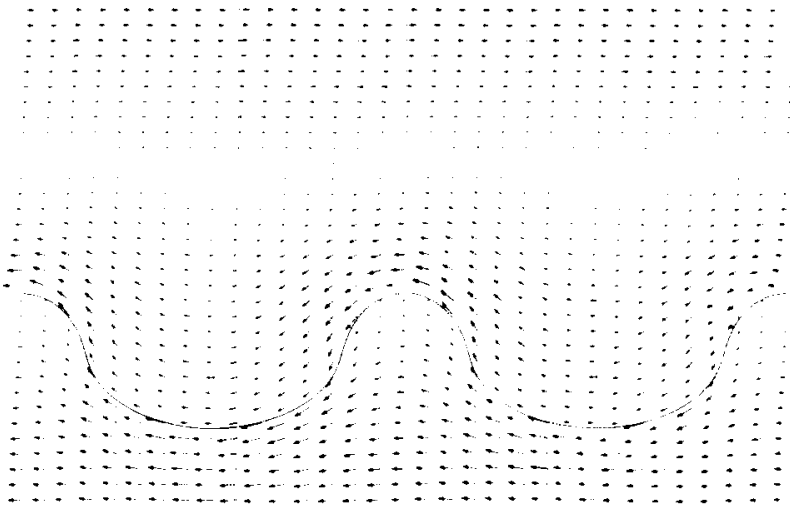

(c)

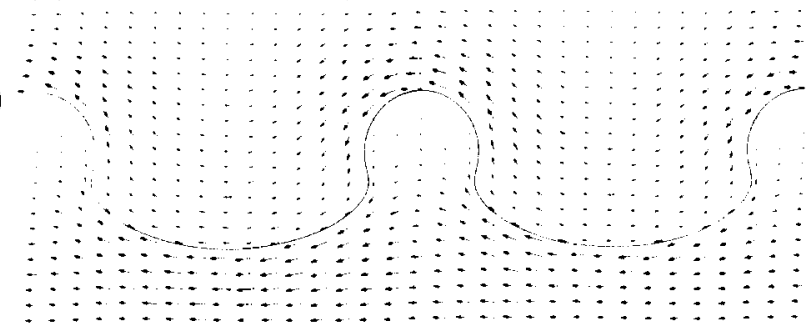

FIG. 8. Near interface velocity field in reference frame at rest with respect to the wave, $D \rightarrow \infty, \Omega=-2 / \pi$, upper branch; (a) $\delta / \pi=0.10$, (b) $\delta /$ $\pi=0.175,(\mathrm{c}) \delta / \pi=0.205$

original reference frame. This differs slightly from the suggestion of Holyer $^{5}$ that $\delta_{\max }$ coincides with infinite wave slope where the local horizontal fluid speed equals the wave speed. At least for these Boussinesq waves it is evident that $\delta_{\max }$ does not necessarily coincide with infinite wave slope. In their study of nonlinear interfacial waves on a vortex sheet, Saffman and Yuen ${ }^{4}$ conjecture that the highest wave is associated with extremely sharp curvatures appearing at either the crest or trough. In some special circumstances, they found solutions of this kind in which one fluid is stagnant and the wave is essentially that of the free-surface Stokes wave, which is known to reach its maximum amplitude when the wave has a corner at the crest. In I (where $U_{1} \neq 0, U_{2}=0, \Omega_{1}=\Omega_{2}=0$ ) we found one instance of a highest wave of this kind (for $U_{1}=0.2, D / \pi=0.01$, lower branch), but in most cases the highest wave was apparently 


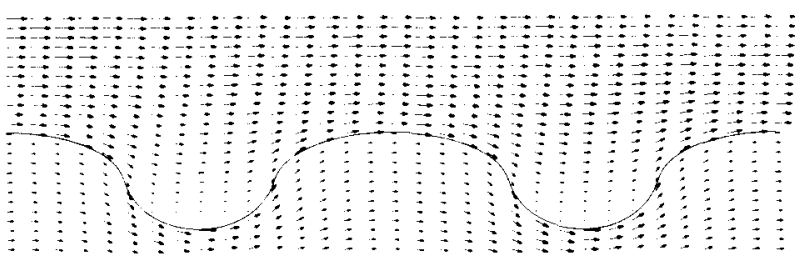

FIG. 9. Near interface velocity field in reference frame at rest with respect to the wave, $D \rightarrow \infty, \Omega=-2 \pi$, lower branch; $\delta / \pi=0.125$.

associated either with the kinematic condition that a stagnation point appears on the profile or with a geometrical constraint associated with the upper boundary. In this paper where $\Omega \neq 0$, there is no solution in which the upper fluid is totally stagnant; and although there may be a solution in which the lower fluid is totally stagnant, we found no evidence of it. In general, if there is a solution with a corner at the crest, then a local analysis similar to that for the freesurface Stokes waves shows that the corner angle should be $120^{\circ}$ and that the flow in the opposing fluid (into which the corner points) should be locally stagnant. This is consistent with the flow field of Fig. 10 which indicates that the cusped highest wave is forming when the wave crest touches the velocity field stagnation point formed in the disturbed critical layer $(Y=|C / \Omega|$ for the unperturbed flow). Thus there will be a region of $D-\Omega$ space where $\Omega$ is large enough to generate a cusped limiting solution, but we have not presently attempted the very extensive calculations required to define the appropriate bounding curves.

The upper branch velocity field of Figs. 8, 10, and 12 show the presence of closed regions of rotational flow (the "cats eyes" pattern) positioned at about the critical layer of the undisturbed flow, which convect with the wave. In Figs. 12 (b) and 12(c), separation/reattachment points on the upper rigid surface can be seen bounding the closed "separation bubble" but in the weaker shear case of Fig. 11 no recirculating region occurs. These velocity fields for finite $D$ are thus rather similar kinematically to those obtained by Perry and Fairlie $^{6}$ in their study of a separation bubble in a turbulent boundary layer.

The present results may be used to estimate the deflection of the free-surface $Y=D$ in the case of small but finite $\alpha$. Taking for simplicity $\omega_{1}=0$ (the results will be unchanged by finite shear) we may write the dimensionless Bernoulli equation (6) in the upper layer in a reference frame at rest with respect to the wave as

$$
\begin{aligned}
\alpha P_{1}= & -(1-\alpha)\left[\frac{1}{2} \alpha\left(U^{2}+V^{2}\right)+Y\right] \\
& +\alpha(1-\alpha)\left(\Lambda_{1}+\frac{1}{2} C^{2}\right),
\end{aligned}
$$

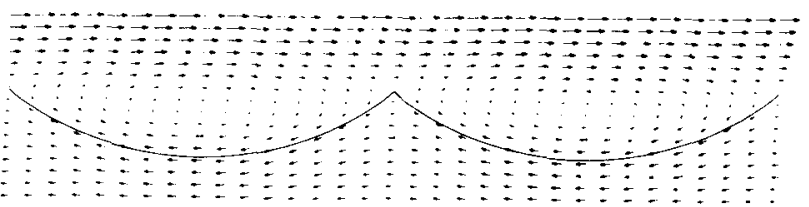

FIG. 10. Near interface velocity field in reference frame at rest with respect to the wave, $D \rightarrow \infty, \Omega=-100 / \pi$, upper branch; $\delta / \pi=0.0875$. (a)

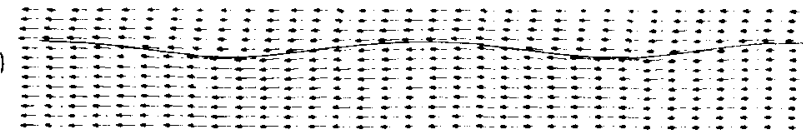

(b)

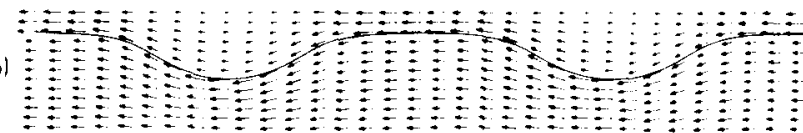

(c)

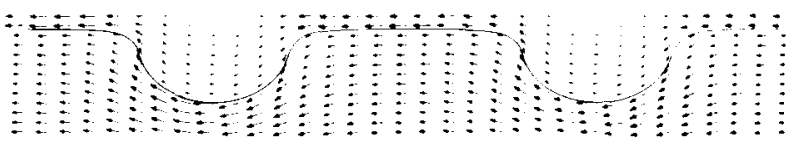

FIG. 11, Near interface velocity field in reference frame at rest with respect to the wave, $D / \pi=0.1, \Omega=-2 / \pi$, upper branch; (a) $\delta / \pi=0.02$, (b) $\delta /$ $\pi=0.06$, (c) $\delta / \pi=0.095$.

where $P_{1}=p_{1} /\left[\frac{1}{2}\left(\rho_{1}+\rho_{2}\right) \alpha g(\lambda / \pi)\right]$. Applying $(21)$ to $Y=D$ with $p_{1}=-\rho_{1} g d_{1}\left[\alpha P_{1}=-(1-\alpha) D\right]$ we may expand other quantities in (21) on $Y=D$ as

$$
\begin{aligned}
& U-i V=U^{(0)}-i V^{(0)}+O(\alpha), \\
& Y=D+\alpha Y^{(1)}, \\
& \Lambda_{1}=\Lambda_{1}^{(0)}+O(\alpha), \\
& C=C^{(0)}+O(\alpha),
\end{aligned}
$$

where the $(0)$ superscript refers to the present results with a rigid upper surface and $Y^{(1)}$ is the order $(\alpha)$ correction to the surface shape. Substituting (22) into (21), equating order $(\alpha)$ terms and using $(25 b)$ of Sec. $V$ to estimate the magnitude of the velocity, we find that

$$
Y^{(1)}=O\left[C^{(0)}\left(\Lambda_{1}^{(0)}\right)^{1 / 2}\right] .
$$

From the present results the right side of $(23)$ is always of order 1 or less and since the most thermocline applications $\alpha \sim O\left(10^{-3}\right)$ (see Grimshaw ${ }^{7}$ ), the free surface elevation correction is thus genuinely small in the case of physical interest even for very-high-amplitude waves.

\section{INTEGRAL PROPERTIES}

\section{A. Analysis}

Once analytical or numerical solutions are obtained the various integral properties may be readily calculated. The

(a)

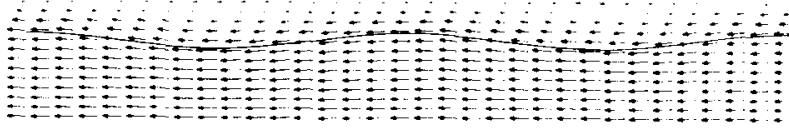

(b)

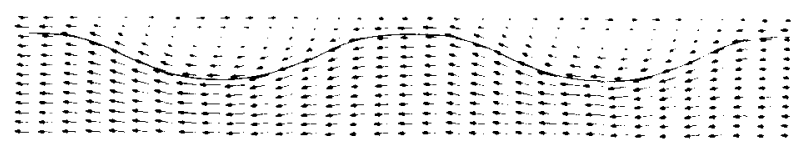

(c)

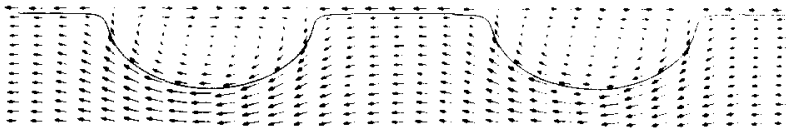

FIG. 12. Near interface velocity field in reference frame at rest with respect to the wave, $D / \pi=0.1, \Omega=-10 / \pi$, upper branch; (a) $\delta / \pi=0.02$, (b) $\delta /$ $\pi=0.06$, and $(\mathrm{c}) \delta / \pi=0.10$. 
most relevant of these are the excess impulse $I$ due to the presence of the wave, the excess kinetic and potential energies per wavelength $T$ and $V$, respectively, the excess flux of $x$-momentum (radiation stress) $S_{x}$ and the excess energy flux in the $x$-direction $F$. Returning to dimensional coordinates and using (4), $I$ may be written as

$$
I=\rho_{2} Q_{2}-\rho_{1} Q_{1} \text {. }
$$

For the basic flow (1) the other quantities may be defined as

$$
\begin{aligned}
T= & \left\langle\frac{1}{2} \rho_{2} \int_{-d_{2}}^{\eta}\left(u_{2}^{2}+v_{2}^{2}\right) d y+\frac{1}{2} \rho_{1} \int_{\eta}^{d_{1}}\left(u_{1}^{2}+v_{1}^{2}\right) d y\right\rangle \\
& -\frac{1}{2} \rho_{2} \bar{u}_{2}^{2} d_{2}-\frac{1}{2} \rho_{1} \int_{0}^{d_{1}}\left(\bar{u}_{1}-\omega_{1} y\right)^{2} d y \\
V=\frac{1}{2} & g\left(\rho_{2}-\rho_{1}\right)\left\langle\eta^{2}\right\rangle \\
S= & \left\langle\int_{-d_{2}}^{\eta}\left(p_{2}+\rho_{2} u_{2}^{2}\right) d y+\int_{\eta}^{d_{1}}\left(p_{1}+\rho_{1} u_{1}^{2}\right) d y\right) \\
& -\frac{1}{2} g\left(\rho_{2} d_{2}^{2}-\rho_{1} d_{1}^{2}\right)-\rho_{2} \bar{u}_{2}^{2} d_{2} \\
& -\rho_{1} \int_{0}^{d_{1}}\left(\bar{u}_{1}-\omega_{1} y\right)^{2} d y \\
F= & \left\langle\int_{-d_{2}}^{\eta}\left(p_{2}+\rho_{2} g y+\frac{1}{2} \rho_{2}\left(u_{2}^{2}+v_{2}^{2}\right)\right) u_{2} d y\right. \\
& \left.+\int_{\eta}^{d_{1}}\left(p_{1}+\rho_{1} g y+\frac{1}{2} \rho_{2}\left(u_{1}^{2}+v_{1}^{2}\right)\right) u_{1} d y\right\rangle \\
& -\frac{1}{2} \rho_{2} d_{2} \bar{u}_{2}^{3}-\frac{1}{2} \rho_{1} \int_{0}^{d}\left(\bar{u}_{1}-\omega_{1} y\right)^{3} d y
\end{aligned}
$$

Not all of these quantities are independent and relations amongst them may be obtained. Introducing \langle\rangle$_{\eta}$ as in (5) but with the integrand evaluated on $y=\eta$ we first find that use of the $y$-momentum equation leads to

$$
\begin{aligned}
& \left\langle p_{2} / \rho_{2}\right\rangle_{\eta}=0=L_{2}-\frac{1}{2}\left\langle\hat{u}_{2}^{2}\left(-d_{2}\right)\right\rangle, \\
& \left\langle p_{1} / \rho_{1}\right\rangle_{\eta}=0=L_{1}-\frac{1}{2}\left(\hat{u}_{1}^{2}\left(d_{1}\right)\right\rangle,
\end{aligned}
$$

where we have normalized such that $\left\langle p_{1}\right\rangle_{\eta}=\left\langle p_{1}\right\rangle_{\eta_{j}}=0$. Note that for $d_{2} \rightarrow \infty, L_{2} \rightarrow 0$. Use of $(3)-(6)$ and (24)-(25) then leads to, after considerable algebra,

$$
\begin{aligned}
2 T= & c\left(\rho_{2} Q_{2}-\rho_{1} Q_{1}\right)-\rho_{1} \bar{u}_{1} Q_{1}+\rho_{2} \bar{u}_{2} Q_{2}-\rho_{1} \omega_{1} H \\
S= & 4 T-3 V+2 \rho_{1} \bar{u}_{1} Q_{1}-2 \rho_{2} \bar{u}_{2} Q_{2}+\rho_{1} d_{1} L_{1}+\rho_{2} d_{2} L_{2} \\
& +\frac{1}{2} \rho_{2} d_{2}\left\langle\hat{u}_{2}^{2}\left(-d_{2}\right)\right\rangle+\frac{1}{2} \rho_{1} d_{1}\left\langle\hat{u}_{1}^{2}\left(d_{1}\right)\right\rangle+4 \rho_{1} \omega_{1} H,
\end{aligned}
$$$$
F=c\left[3 T-2 V+2 \rho_{1} \vec{u}_{1} Q_{1}-2 \rho_{2} \bar{u}_{2} Q_{2}+3 \omega_{1} H\right.
$$$$
\left.+\frac{1}{2} \rho_{2} d_{2}\left\langle\hat{u}_{2}^{2}\left(-d_{2}\right)\right\rangle+\frac{1}{2} \rho_{1} d_{1}\left\langle\hat{u}_{1}^{2}\left(d_{1}\right)\right\rangle\right]
$$$$
+\rho_{2} L_{2}\left(Q_{2}+d_{2} \bar{u}_{2}\right)+\rho_{1} L_{1}\left(Q_{1}+\bar{u}_{1} d_{1}-\frac{1}{2} \omega_{1} d^{2}\right)
$$$$
+\frac{1}{2} \rho_{2} \bar{u}_{2}^{2} Q_{2}-\frac{1}{2} \rho_{1} \bar{u}_{1}^{2} Q_{1}
$$$$
+\frac{1}{2} \rho_{1} \omega_{1} \bar{u}_{1}^{2}\left\langle\eta^{2}\right\rangle+\frac{1}{2} \omega_{1} \rho_{1} Q_{1}^{2} \text {, }
$$

where

$$
\begin{aligned}
H & =\left\langle\int_{\eta}^{d_{1}} y(u-c) d y\right\rangle+\frac{1}{2}(c-\bar{u}) d^{2}+\frac{1}{3} \omega_{1} d^{2} \\
& =\frac{1}{2}\left(c-\bar{u}_{1}\right)\left\langle\eta^{2}\right\rangle+\frac{1}{3} \omega_{1}\left\langle\eta^{3}\right\rangle+\left\langle\hat{\phi}_{1} \eta \eta_{x}\right\rangle_{\eta}
\end{aligned}
$$

For $d_{2} \rightarrow \infty, \bar{u}_{2}=\bar{u}_{1}=0$, results from the second-order Stokes expansion may be obtained using (4) and (24) together with (12)-(13). The principal integral parameters [including wave speed correction $c-c_{0}$ where $c_{0}$ is given by (11)] are then to order $\Delta^{2}$,

$$
c-c_{0}=(k \Delta / 2)^{2} c_{1},
$$

where

$$
\begin{aligned}
c_{1}= & k\left[2 F^{2} G+2 c_{0}^{2}\left[1+S_{1}\left(2-S_{1}^{2}\right)\right]\right. \\
& \left.-\frac{\omega_{1}}{k}\left(2 c_{0}\left(S_{1}^{2}-1\right)+\frac{\omega_{1}}{2 k} \frac{\left(S_{1}^{2}+1\right)}{S_{1}}\right)\right] \\
& \times\left[2 k c_{0}\left(1+S_{1}\right)+\omega_{1}\right]^{-1}, \\
Q_{1}= & -\left(\frac{k \Delta}{2}\right)^{2}\left(\frac{2 c_{0} S_{1}}{k}+\frac{\omega_{1}}{k^{2}}\right), \\
Q_{2}= & -\frac{c_{0}}{k}\left(\frac{k \Delta}{2}\right)^{2}, \\
T= & \frac{c_{0}^{2}}{2}\left(\frac{k \Delta}{2}\right)^{2}\left(1+S_{1}\right), \\
V= & g\left(\rho_{2}-\rho_{1}\right)\left(\Delta^{2} / 4\right), \\
L_{1}= & \left(\frac{k \Delta}{2}\right)^{2} \frac{c_{0}^{2}}{\sinh ^{2}\left(k d_{1}\right)} .
\end{aligned}
$$

For the numerical solutions the single parameter $L_{1}-\omega_{1} Q_{1}$ is obtained directly. The other independent quantities in (4) and (26), namely $Q_{1}, Q_{2},\left\langle\eta^{2}\right\rangle,\left\langle\eta^{3}\right\rangle$, and $\left\langle\hat{\phi}_{1} \eta \eta_{x}\right\rangle_{\eta}$ may be easily expressed in terms of quantities that can be calculated from the shape of and the velocity field on the wave profile. Thus $L_{1}, V$ [from (24c)], and $T, S, F$ [from (26)] can be readily determined. The integral properties so calculated showed similar trends to those reported in I. Figure 13 shows an example of some integral properties in dimensionless form for finite $D$, compared to the predictions of (27). No maxima occur, the principal trend being one of monotonic variation with increasing $\delta / \pi$. Detailed results from other cases may be obtained from the first author.

\section{CONCLUSIONS}

We have studied the effect of a constant vorticity upper layer on the characteristics of progressive nonlinear waves in

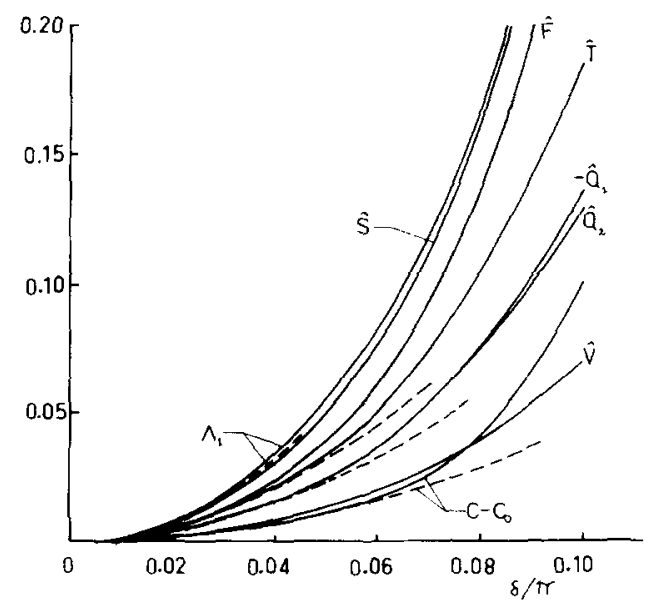

FIG. 13. Integral properties of waves, $D / \pi=0.1, \Omega=-10 / \pi$ (upper branch); - numerical; - - - selected second-order Stokes expansions. 
a two-layer Boussinesq fluid bounded by an upper rigid surface. Although the highest wave appeared in a variety of configurations, for all cases the approach to maximum amplitude was found to be consistent with the appearance of a stagnation point on the wave profile in the reference frame at rest with respect to the wave. Higher amplitudes would then imply locally reversed flow on the interface profile which is presumably either kinematically impossible under present symmetry assumptions or requires a different wave topology, for example a multiply-connected vortex-sheet configuration with disconnected lobes.

The appeal of the present model is that it allows realistic upper-layer shear but remains stable to infinitesimal interface disturbances. This of course does not imply that finiteamplitude solutions will be likewise stable. Indeed, in view of regions of strong vortex sheet strength which can be seen along the interface in the velocity fields of Figs. 7-12, it seems likely that some form of local Kelvin-Helmholtz stability will appear at sufficiently high wave amplitude. A finite-amplitude stability analysis of the present solutions is thus necessary to define the stability-amplitude envelope and to determine the dominant instability mechanism, if present at high amplitude. This is currently the subject of a further study.

'D. I. Pullin and R. H. J. Grimshaw, Phys. Fluids 26, 897 (1983).

${ }^{2}$ D. I. Pullin, J. Fluid Mech. 106, 401 (1981).

${ }^{3}$ P. G. Saffman and J. C. Schatzman, SIAM, J. Sci. Stat. Comput. 2, 285 (1981).

${ }^{4}$ P. G. Saffman and H. C. Yuen, J. Fluid Mech. 123, 459 (1982).

${ }^{5}$ J. Y. Holyer, J. Fluid Mech. 93, 433 (1979).

${ }^{6}$ A. E. Perry and D. B. Fairlie, J. Fluid Mech. 69, 657 (1975).

${ }^{7}$ R. J. H. Grimshaw, J.Fluid Mech. 86, 415 (1978). 